\title{
Passivity Based Power Control of Three-Phase Three-Switch Vienna Rectifier
}

\author{
Zhai Dandan ${ }^{1, a}$, Wang Jiuhe ${ }^{2, b}$ and She Dongjin ${ }^{3, c}$ \\ ${ }^{1}$ School of Automation, Beijing Information Science \& Technology University, Beijing, China \\ ${ }^{2}$ School of Automation, Beijing Information Science \& Technology University, Beijing, China \\ ${ }^{3}$ School of Automation, Beijing Information Science \& Technology University, Beijing, China \\ azhaidandan_happy@126.com, bwjhyhrwm@163.com, cjade_1228@163.com
}

Keywords: Vienna Rectifier, EL model, Passivity based power control, Damping injecting.

\begin{abstract}
Power Euler-Lagrange(EL) mathematical model of three-phase three-switch Vienna rectifier is set up in synchronous rotation $d q$ coordinate system based on its topological structure. According to power EL mathematical model and passivity of Vienna rectifier, passivity based power controller is designed by damping injecting. The passivity based power controller can make power error storage function to zero quickly, so that desired equilibrium point, such as unity power factor, low AC current harmonic and constant DC voltage is realized. Dynamic decoupling between active and reactive power is obtained because of passivity based power controller, and Vienna rectifier possess good dynamic and stable performances. Simulation results under different load show that passivity based power control of Vienna rectifier is feasible.
\end{abstract}

\section{Introduction}

Three-level Vienna rectifier is characterized by low switching frequency, high input power factor, low device voltage stress, high power density, sinusoidal input current, high reliability and small size inductance, etc, which make it a suitable topology for medium- and high-power applications with high power density and new energy fields ${ }^{[1]}$. Simultaneously, Vienna rectifier could still work under unbalanced power grid and default phase, which has increasingly aroused great concern among home and overseas scholars. If the topological structure is known, performance largely depends on control strategy and PWM modulation method. Due to it, control strategies become a interested topic in the study of Vienna rectifier. Passive control theory ${ }^{[2]}$ start with system energy, study how to control system energy and achieve the control purpose. Then it is feasible to achieve global stability without singularity problem. This method has strong robustness for varying parameters and external disturbance, and it is a nonlinear control method in nature. So Vienna rectifier control strategy based on passive control was researched in [3]. This paper studies three-switch Vienna rectifier, and passivity based power controller is proposed for the first time, which can realize dynamic decoupling between active power and reactive power and obtain an excellent dynamic and stable performance. Passivity based power control strategy of three-phase three-switch Vienna rectifier can be expanded to other types Vienna rectifiers.

\section{Circuit topology and switch state of Vienna rectifier}

The power circuit topology of three-phase three-switch Vienna rectifier is shown in Fig.1. For example, if the switch $T_{a}\left(T_{a}=1\right)$ is on, phase leg A will be clamped to the dc-link neutral point. If $o$ is the reference point, $u_{a o}$ will be 0 . If $T_{a}\left(T_{a}=0\right)$ is off and the phase current $i_{a}$ is positive, $u_{a o}$ will be $\mathrm{u}_{\mathrm{C} 1}$. Similarly, if $T_{a}\left(T_{a}=0\right)$ is off and $i_{a}$ is negative, $u_{a o}$ will be $-u_{C 2}$.Phase leg A can get three levels such as $u_{C l} 、 0 、-u_{C 2}$, The same operation principle applies to phase $\mathrm{B}$ and phase $\mathrm{C}$. 


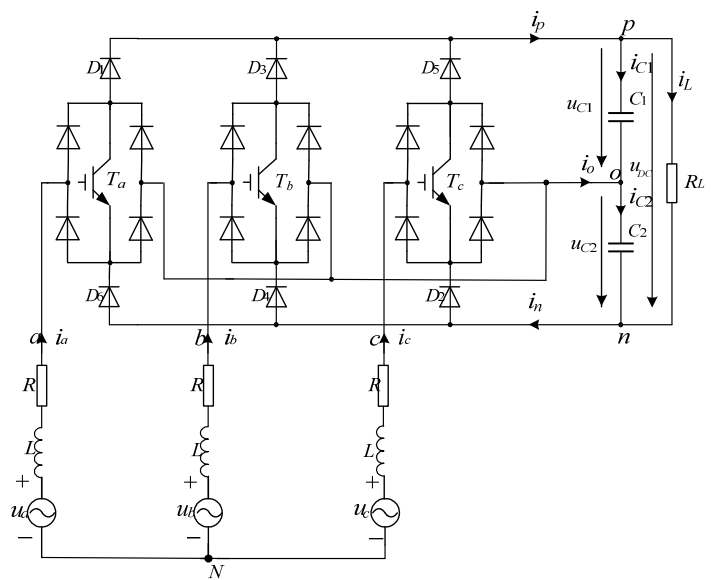

Fig.1 Power circuit of Vienna rectifier

The switch function of VIENNA rectifier is provided as follows:

$$
S_{k}=\left\{\begin{array}{rl}
+1 & T_{k}=0, i_{k}>0 \\
0 & T_{k}=1 \\
-1 & T_{k}=0, i_{k}<0
\end{array} \quad k=a, b, c\right.
$$

\section{Power mathematical model of Vienna rectifier}

The Mathematical Model of the System. Considering that three-phase power supply are balanced, the mathematical model of Vienna rectifier in the three-phase $a b c$ coordinates has been discussed in [3] and [4], and its model is very complex. To simplify the model and its controller design, mathematical model in the three-phase $a b c$ coordinates can be written as

$$
\left\{\begin{array}{l}
L \frac{\mathrm{d} i_{a}}{\mathrm{~d} t}+R i_{a}+\frac{1}{2} S_{a} u_{D C}\left[1+\operatorname{sgin}\left(i_{a}\right) \frac{\Delta u_{D C}}{u_{D C}}\right]-u_{N O}=u_{a} \\
L \frac{\mathrm{d} i_{b}}{\mathrm{~d} t}+R i_{b}+\frac{1}{2} S_{b} u_{D C}\left[1+\operatorname{sgin}\left(i_{b}\right) \frac{\Delta u_{D C}}{u_{D C}}\right]-u_{N O}=u_{b} \\
L \frac{\mathrm{d} i_{c}}{\mathrm{~d} t}+R i_{c}+\frac{1}{2} S_{c} u_{D C}\left[1+\operatorname{sgin}\left(i_{\mathrm{c}}\right) \frac{\Delta u_{D C}}{u_{D C}}\right]-u_{N O}=u_{c} \\
C \frac{\mathrm{d} u_{D C}}{\mathrm{~d} t}+\frac{2 u_{D C}}{R_{L}}-S_{a} i_{a}-S_{b} i_{b}-S_{c} i_{c}=0 \\
C \frac{\mathrm{d} \Delta u_{D C}}{\mathrm{~d} t}=S_{a} i_{a} \sin \left(i_{\mathrm{a}}\right)+S_{b} i_{b} \operatorname{sgin}\left(i_{\mathrm{b}}\right)+S_{c} i_{c} \operatorname{sgin}\left(i_{\mathrm{c}}\right)
\end{array}\right.
$$

Where $u_{N O}$ is the voltage across the neutral point of the dc-link and the neutral point of the three-phase input voltage; When $i_{k}>0, \operatorname{sgin}\left(i_{k}\right)=1 ; \quad i_{k}<0, \operatorname{sgin}\left(i_{k}\right)=-1, k=a, b, c ; \quad \Delta u_{D C}=u_{C l}-u_{C 2}$.

Define $d_{k}=S_{k}\left[1+\frac{\Delta u_{D C}}{u_{D C}} \operatorname{sign}\left(i_{k}\right)\right]$.as a new switch function, considering that $\frac{\Delta u_{D C}}{u_{D C}} \square 1$, the following transformation is proposed:

$$
\left\{\begin{array}{l}
d_{k}\left[1-\frac{\Delta u_{D C}}{u_{D C}} \operatorname{sign}\left(i_{k}\right)\right] \approx S_{k} \\
d_{k}\left[\operatorname{sign}\left(i_{k}\right)-\frac{\Delta u_{D C}}{u_{D C}}\right] \approx S_{k} \operatorname{sign}\left(i_{k}\right)
\end{array}\right.
$$

Such that, substitute the formula (3) into formula (2), as follows 


$$
\left\{\begin{array}{l}
L \frac{\mathrm{d} i_{a}}{\mathrm{~d} t}+R i_{a}+\frac{1}{2} d_{a} u_{D C}-u_{N O}=u_{a} \\
L \frac{\mathrm{d} i_{b}}{\mathrm{~d} t}+R i_{b}+\frac{1}{2} d_{b} u_{D C}-u_{N O}=u_{b} \\
L \frac{\mathrm{d} i_{c}}{\mathrm{~d} t}+R i_{c}+\frac{1}{2} d_{c} u_{D C}-u_{N O}=u_{c} \\
C \frac{\mathrm{d} u_{D C}}{\mathrm{~d} t}+\frac{2 u_{D C}}{R_{L}}=\sum_{k=a, b, c} d_{k}\left[1-\frac{\Delta u_{D C}}{u_{D C}} \operatorname{sign}\left(i_{k}\right)\right] i_{k} \\
C \frac{\mathrm{d} \Delta u_{D C}}{\mathrm{~d} t}=\sum_{k=a, b, c} d_{k}\left[\operatorname{sign}\left(i_{k}\right)-\frac{\Delta u_{D C}}{u_{D C}}\right] i_{k}
\end{array}\right.
$$

Transform the mathematical model of the $a b c$ coordinate frame into a two-phase synchronous rotating $d-q$ coordinate frame, the $d-q$ mathematical model can be described as

$$
\left\{\begin{array}{l}
L \frac{\mathrm{d} i_{d}}{d t}-\omega L i_{q}+R i_{d}+\frac{1}{2} d_{d} u_{D C}=u_{d} \\
L \frac{\mathrm{d} i_{q}}{d t}+\omega L i_{d}+R i_{q}+\frac{1}{2} d_{q} u_{D C}=u_{q} \\
C \frac{\mathrm{d} u_{D C}}{d t}+\frac{2 u_{D C}}{R_{L}}=\frac{3}{2}\left(d_{d} i_{d}+d_{q} i_{q}\right) \\
C \frac{\mathrm{d} \Delta u_{D C}}{d t}=\alpha d_{0} i_{d}
\end{array}\right.
$$

Where $\alpha=2 / \pi, \mathrm{i}_{d} 、 \mathrm{i}_{q}$ are the currents on the $d$ axis and $q$ axis respectively; $u_{d}, u_{q}$ are the voltage on the $d$ axis and $q$ axis respectively; $d_{d} 、 d_{q} 、 d_{0}$ are components of switching function on $d 、 q$ and 0 axis respectively.

Power mathematical model. Under three-phase balanced power supply, the instantaneous active power is $p=1.5 u_{d} i_{d}$, reactive power is $q=1.5 u_{d} i_{q}$. Thus power mathematical model can be get as follows which use $p 、 q 、 u_{D C} 、 \Delta u_{D C}$ as state-variable.

$$
\left\{\begin{array}{l}
\frac{2}{3} L \frac{\mathrm{d} p}{\mathrm{~d} t}-\frac{2}{3} \omega L q+\frac{2}{3} R p+u_{d} d_{d} \frac{u_{D C}}{2}=u_{d}^{2} \\
\frac{2}{3} L \frac{\mathrm{d} q}{\mathrm{~d} t}+\frac{2}{3} \omega L p+\frac{2}{3} R q+u_{d} d_{q} \frac{u_{D C}}{2}=u_{d} u_{q} \\
\frac{u_{d}^{2} C}{2} \frac{\mathrm{d} u_{D C}}{\mathrm{~d} t}+\frac{u_{d}^{2} u_{D C}}{R_{L}}-\frac{1}{2} u_{d} d_{d} p-\frac{1}{2} u_{d} d_{q} q=0 \\
\frac{u_{d}^{2} C}{2} \frac{\mathrm{d} \Delta u_{D C}}{\mathrm{~d} t}=d_{0} \frac{\alpha p u_{d}}{3}
\end{array}\right.
$$

Substitute the formula (6) into power EL form

$$
\boldsymbol{M} \dot{\boldsymbol{x}}+\boldsymbol{J} \boldsymbol{x}+\boldsymbol{R} \boldsymbol{x}=\boldsymbol{u}
$$

Where

$$
\begin{aligned}
& \boldsymbol{M}=\left(\begin{array}{cccc}
\frac{2}{3} L & 0 & 0 & 0 \\
0 & \frac{2}{3} \mathrm{~L} & 0 & 0 \\
0 & 0 & \frac{u_{d}^{2} L}{2} & 0 \\
0 & 0 & 0 & \frac{u_{d}^{2} C}{2}
\end{array}\right), \boldsymbol{J}=\left(\begin{array}{cccc}
0 & -\frac{2}{3} \omega L & \frac{u_{d} d_{d}}{2} & 0 \\
\frac{2}{3} \omega L & 0 & \frac{u_{d} d_{q}}{2} & 0 \\
-\frac{u_{d} d_{d}}{2} & -\frac{u_{d} d_{q}}{2} & 0 & 0 \\
0 & 0 & 0 & 0
\end{array}\right), \boldsymbol{R}=\left(\begin{array}{cccc}
\frac{2 R}{3} & 0 & 0 & 0 \\
0 & \frac{2 R}{3} & 0 & 0 \\
0 & 0 & \frac{u_{d}^{2}}{R_{L}} & 0 \\
0 & 0 & 0 & 0
\end{array}\right) \\
& \boldsymbol{x}=\left(\begin{array}{lllll}
p & q & u_{\mathrm{DC}} & \Delta u_{\mathrm{DC}}
\end{array}\right)^{T}, \boldsymbol{J}=-\boldsymbol{J}^{\mathrm{T}}, \boldsymbol{u}=\left(\begin{array}{llll}
u_{d}^{2} & u_{d} u_{q} & 0 & d_{0} \alpha p u_{d} / 3
\end{array}\right)^{T}
\end{aligned}
$$

\section{The Design of Passivity based power Controller}

The Desired Point of Stable Equilibrium. Set the desired equilibrium point are $\boldsymbol{x}^{*}$, In order to meet unit power factor and low alternating current harmonic, $p \rightarrow p^{*} 、 q \rightarrow q^{*}=0$ and $u_{D C}$ should be 
stable in the desired value $u_{D C}{ }^{*}\left(u_{D C}{ }^{*}>\sqrt{3} u_{\mathrm{d}}\right)$. Substitute these values into equation $(6), p^{*}$ can be described as

$$
p^{*}=\frac{3}{4} \frac{u_{d}^{2}}{R}-\sqrt{\left(\frac{3}{4} \frac{u_{d}^{2}}{R}\right)^{2}-\frac{3 u_{d}^{2} u_{D C}^{* 2}}{2 R_{L} R}}
$$

where $R_{L}=\frac{u_{D C}}{i_{L}}$.

The Design of power Controller. Set $\boldsymbol{x}_{e}=\boldsymbol{x}^{-\boldsymbol{x}^{*}}$ and the error storage function as $H_{e}(\boldsymbol{x})=\frac{1}{2} \boldsymbol{x}_{e}^{T} \boldsymbol{M} \boldsymbol{x}_{e}$.In order to accelerate the error energy to zero, the damping $\boldsymbol{R}_{a}=\operatorname{diag}\left(r_{a 1} \quad r_{a 2} \quad r_{a 3} \quad r_{a 4}\right)$ is inject into the system, set $\boldsymbol{R}_{d} \boldsymbol{x}_{e}=\left(\boldsymbol{R}+\boldsymbol{R}_{a}\right) \boldsymbol{x}_{e}$, the formula (7) can be described as

$$
\boldsymbol{M} \dot{\boldsymbol{x}}_{e}+\boldsymbol{R}_{d} \boldsymbol{x}_{e}=\boldsymbol{u}-\left(\boldsymbol{M} \dot{\boldsymbol{x}}^{*}+\boldsymbol{J} \boldsymbol{X}+\boldsymbol{R} \boldsymbol{x}^{*}-\boldsymbol{R}_{a} \boldsymbol{x}_{e}\right)
$$

The passive-based controller ${ }^{[5]}$ can be written as

$$
\boldsymbol{u}=\boldsymbol{M} \dot{\boldsymbol{x}}^{*}+\boldsymbol{J}_{\boldsymbol{X}}+\boldsymbol{R} \boldsymbol{x}^{*}-\boldsymbol{R}_{a} \boldsymbol{x}_{e}
$$

Considering the formula (10) and $\boldsymbol{M x}^{*}=0$, then we can get the switch function corresponding to power controller.

$$
\left\{\begin{array}{l}
d_{d}=\frac{2\left[3 u_{d}^{2}+2 \omega L q-2 R p^{*}+3 r_{a 1}\left(p-p^{*}\right)\right]}{3 u_{d} u_{D C}} \\
d_{q}=\frac{2\left[-2 \omega L p+3 r_{a 2} q\right]}{3 u_{d} u_{D C}} \\
d_{0}=\frac{-3 r_{a 4} \Delta u_{D C}}{\alpha p u_{d}}
\end{array}\right.
$$

Combined with the power controller, we get the overall control diagram in Fig. 2 of the control system:

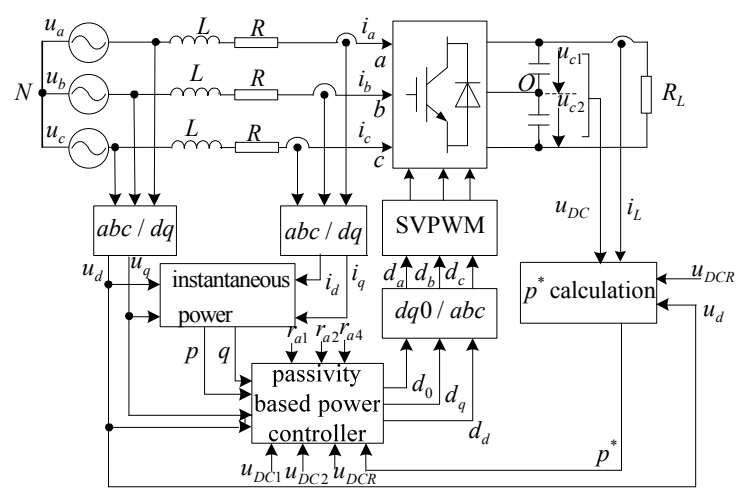

Fig.2 Control block diagram of Vienna rectifier

\section{Simulation results and discussion}

The system is simulated in MATLAB/simulink environment.According to Fig.2, formula (8) and formula (11), we establish the Vienna rectifier passive power control model. Simulation parameters are shown in table 1.

Table1 simulation parameter

\begin{tabular}{|l|l|}
\hline parameters & numerical value \\
\hline grid RMS voltage $U$ & $220[\mathrm{~V}]$ \\
\hline frequency $f$ & $50[\mathrm{~Hz}]$ \\
\hline DC voltage $U_{D C}{ }^{*}$ & $700[\mathrm{~V}]$ \\
\hline output capacitor $C$ & $2200[\mu \mathrm{F}]$ \\
\hline input inductance $L$ & $5[\mathrm{mH}]$ \\
\hline Switching frequency $f_{s}$ & $10[\mathrm{kHz}]$ \\
\hline rated load $R_{L}$ & $50[\Omega]$ \\
\hline
\end{tabular}


Simulation results under $\boldsymbol{R}_{L}=\mathbf{5 0 \Omega}$. To reflect the dynamic response of Vienna rectifier, the simulation is carried on from zero state to steady state. The simulation results is shown in Fig.3.

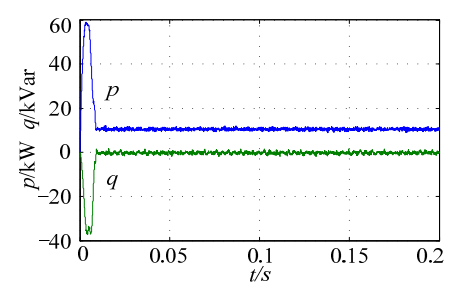

Fig.3 (a) Active $p$ and reactive $q$

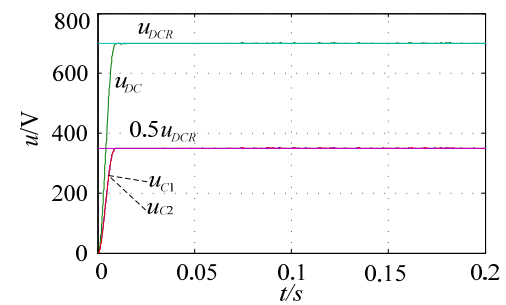

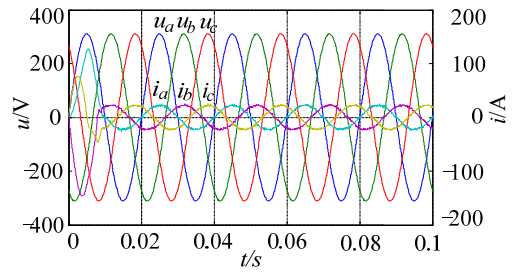

Fig.3 (b) Three-phase AC voltage and current

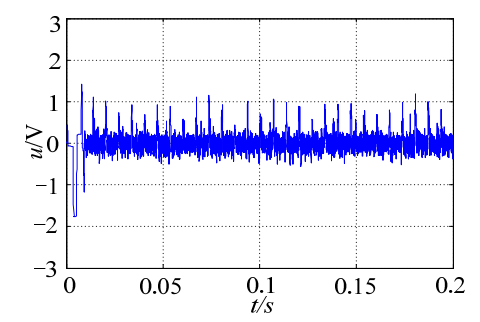

Fig.3 (c) DC desired voltage and output voltage

Fig.3 (d) neutral-point voltage fluctuation

From Fig.3, we observe that DC voltage has entered a steady state at $0.01 \mathrm{~s}$, and startup time of alternating current is $0.007 \mathrm{~s}$. The system maintains the synchronisation of the input current and input voltage in steady state, $\mathrm{THD}=3.26 \%$ and average reactive power to 0 . The system achieve unit power factor, and neutral instantaneous voltage fluctuates around $1 \mathrm{~V}$. These data above suggest that rectifier has reached the expected control goal, and has a good dynamic and stable performance.

Simulation results under $\boldsymbol{R}_{L}=\mathbf{8 0 \Omega}$. In the case of light load (load resistance changed from $50 \Omega$ to $80 \Omega$ at the time of $0.08 \mathrm{~s} \sim 0.1 \mathrm{~s}$ ), simulation results is shown in Fig.4. The Fig. 4 shows that active power fast track the change during light load disturbance. Reactive power, DC voltage has changed little, and the system achieve unit power factor. DC-link voltage balancing is get better.

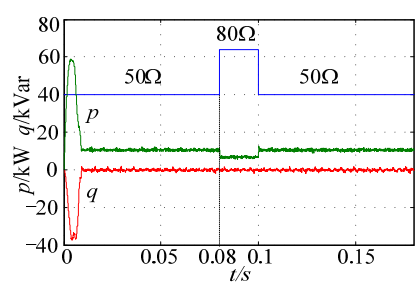

Fig.4 (a) Active p and reactive $\mathrm{q}$

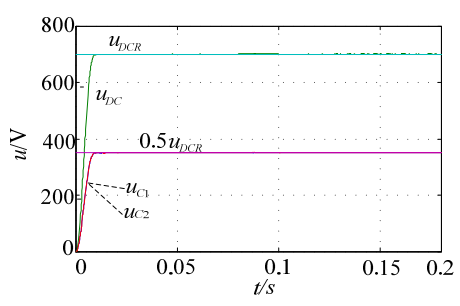

Fig.4 (b) DC desired voltage and output voltage

Simulation results under $\boldsymbol{R}_{L}=\mathbf{2 0 \Omega}$. In the case of overload (load resistance changed from $50 \Omega$ to $20 \Omega$ at the time of $0.08 \mathrm{~s} \sim 0.1 \mathrm{~s}$ ), simulation results is shown in Fig.5. The Fig. 5 shows that active power fast track the change of the load during overload disturbance.Reactive power did not change, and achieve unit power factor. $20 \mathrm{~V}$ fluctuation appear, then stable in $700 \mathrm{~V}$. DC-link voltage balancing is get better.

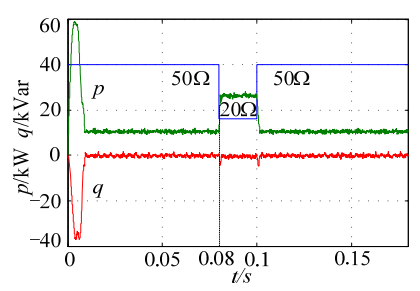

Fig.5 (a) Active $\mathrm{p}$ and reactive $\mathrm{q}$

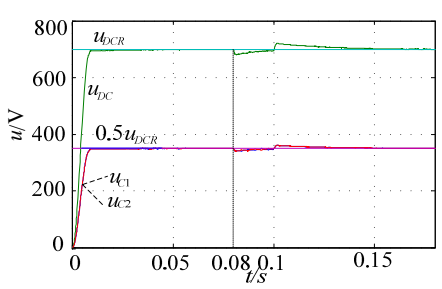

Fig.5 (b) DC desired voltage and output voltage

The simulation results show that passivity based power controller can make the Vienna rectifier obtain a good dynamic and stable performance, especially the introduction of $d_{0}$, obtain a good dc-link voltage balance. 


\section{Acknowledgements}

This work is supported by national natural science foundation of China (51477011)/ by program of Beijing Natural Science Foundation (KZ201511232035)/ Funding Project for Science and technology innovation ability enhancement in Institutions under the Jurisdiction of Beijing Municipality (TJSHG201310772024)/Talents cultivation Program for Excellent Youth of Beijing(CIT\&TCT201304111).

\section{References}

[1] Johann W. Kolar, Thomas Friedli. The Essence of Three-Phase PFC Rectifier Systems-Part I [J]. IEEE Transactions on power electronics, 2013,28(1):176-198.

[2] Wang Jiuhe. Passivity based control theory and its applications[M].Beijing: Publishing House of Electronic Industry, 2010.

[3] Wang Jiuhe. Power converter and its passivity based control [M]. Beijing: Science Press, 2014.

[4] H.kanaan, K.Al-Haddad, F.Fnaiech. Modeling and Control of three-phase/switch/level fixed-frequency PWM rectifier:state-space averaged model[J]. IEEE Proc.-Eletr. Power Appl, 2005,152(3):551-557.

[5] WANG Jiuhe, HUANG Lipei, ZHANG Jinlong, et al. Passive controller for three-phase voltage-source PWM rectifier[J]. Electric Power Automation Equipment, 2008, 28(10): 38-41. 